\title{
Análisis computacional de superficie de respuesta de la transferencia de masa durante la etapa de almacenamiento en la cadena de suministro del Ñame (Dioscorea rotundata)
}

\author{
Sara R. De la Ossa, Anji Y. Novoa, Jhonatan A. Rodríguez-Manrique* y Rafael H. Merlano-Porto \\ Facultad de Ciencias Básicas, Ingeniería y Arquitectura. Corporación Universitaria del Caribe CECAR, \\ Sincelejo, Colombia. (correo-e: sara.delaossa@cecar.edu.co; anji.novoa@cecar.edu.co; jhoejo87@gmail.com; \\ rafael.merlano@cecar.edu.co)
}

* Autor a quien debe ser dirigida la correspondencia

Recibido Mar. 11, 2020; Aceptado May. 8, 2020; Versión final Jul. 12, 2020, Publicado Dic. 2020

\begin{abstract}
Resumen
El objetivo de esta investigación fue analizar mediante herramientas computacionales la superficie de respuesta de la transferencia de masa durante el almacenamiento en la cadena de suministro del ñame (Dioscorea rotundata) tipo exportación. Metodológicamente, muestras de ñame fueron almacenadas a diferentes condiciones de temperatura y de tiempo de almacenamiento. La variable de interés fue la pérdida de peso. Para el tratamiento de los datos, se desarrolló un experimento factorial bajo arreglo completamente al azar y un test de Tukey, por triplicado. Los resultados muestran que el incremento de temperatura y tiempo de almacenamiento trae consigo mayor tasa de transferencia de masa, generando elevadas pérdidas de peso en el tubérculo. Se concluye que mediante el uso de herramientas computacionales fue posible determinar eficientemente el efecto en que los factores estudiados presentan una influencia significativa sobre la pérdida de peso en la estructura porosa del ñame.
\end{abstract}

Palabras clave: almacenamiento; transferencia; masa; superficie de respuesta

\section{Computational surface response analysis of mass transfer during storage in the yam (Dioscorea rotundata) supply chain}

\begin{abstract}
The primary objective of this research study was to analyze the response area of mass transfer during storage in the supply chain of export-type yams (Dioscorea rotundata) by using computer tools. Yam samples were stored at different temperature conditions and storage times. The variable of interest was weight loss. A factorial experiment was developed with a completely random arrangement and a Tukey test, in triplicate. The results show that the increase in temperature and storage time increases the rate of mass transfer, generating high weight losses in the tuber. It is concluded that by using computational tools it was possible to efficiently determine that the factors examined have a significant effect on weight loss in yam porous structures.
\end{abstract}




\section{INTRODUCCIÓN}

El ñame es una planta tropical originaria de los continentes africano y asiático, monocotiledónea, de la familia Dioscoreaceae, perteneciente a la especie de los tubérculos, comúnmente cultivada en el Caribe y Centroamérica (Acevedo et al., 2015). Fisiológicamente, es una planta con enredadera trepadora similar a una cuerda, algunas variedades requieren de soportes verticales donde puedan enrollarse para su pleno desarrollo; su aprovechamiento consiste en que la raíz de la planta es comestible y es muy apetecida por sus efectos nutricionales, fitoterapéuticos y propiedades sensoriales de consumo (Tamiru et al., 2008). El ñame se considera como una excelente fuente alimenticia rica en carbohidratos, aminoácidos esenciales, sales minerales (hierro, calcio y fósforo) y vitaminas (vitamina A, tiamina o vitamina B, vitamina C y vitamina B5) que contribuyen a los requerimientos energéticos y de nutrición de millones de personas en los países en desarrollo (González, 2012).

Actualmente, este tubérculo suele tener diversos usos y aprovechamientos; en aspectos culinarios suele prepararse bajo diversas recetas, la más habitual es ser empleado para el consumo directo posterior a procesos de cocción. También es común utilizar este tubérculo para la elaboración de sopas, obtención de productos fritos tipo snack, fabricación de harinas, postres y tortas (Alvis et al., 2016). Además, se utiliza en algunas industrias para la preparación de papillas para infantes, suele aprovecharse también como materia prima para la elaboración de concentrados para animales a base de proteínas. Adicionalmente, a partir del ñame es usual obtener de almidones nativos y modificados para ser utilizados con fines medicinales en la industria farmacéutica y cosmética (Acosta y Blanco, 2013).

Por otro lado, información de dominio público indica que existen seis géneros de ñame correspondientes con aspectos exógenos como la zona de cultivo, la morfología y la composición que desarrolle. Dentro de estos géneros, se destaca el Dioscorea con 600 especies identificadas, tal como Dioscorea alata (ñame de agua), Dioscorea rotundata (ñame blanco o ñame espino), Dioscórea cayenensis (ñame amarillo), Dioscórea japónica, Dioscórea bulbífera, Dioscórea dodecaneura, Dioscórea zingiberensis, Dioscórea esculenta, Dioscórea polygonoides, Dioscórea trífida, ñame criollo diamante; cuyas características y coloraciones varían entre amarillo, blanco, rosado y purpura teniendo en cuenta la especie de ñame a la que se haga referencia (Reina-Aranza, 2012). La predilección por el ñame como sustento alimentario incentiva el desarrollo y aumento de operaciones de comercio exterior para la economía de los países productores, lo cual es un aspecto beneficioso para los sectores involucrados en su cadena de suministro.

La gran difusión geográfica del tubérculo como insumo de apreciación social y económica, ha incrementado su cultivo nacional e internacional, principalmente en países de Centroamérica y del Caribe (Onyeka et al., 2006). Es así como entre los años 2015 y 2016, Haití, Cuba y Brasil destacaron por su producción del tubérculo de 909.473 ton, 741.358 ton y 499.298 ton, respectivamente. Por su parte, Colombia posee una de las tasas mundiales más significativas de rendimiento en el cultivo de ñame. Según el Instituto Colombiano Agropecuario, en el ñame espino mejorado (Dioscorea rotundata) y el ñame criollo diamante se han experimentado notorios incrementos en la demanda, resaltando los volúmenes de exportación a países como algunas Islas del Caribe, Puerto Rico y Estados Unidos, entre las que se enfatizan las exportaciones a este último territorio (Agronet, 2009).

A pesar de las prometedoras estadísticas de producción de este tubérculo, las características de los tratamientos efectuados en las etapas de transporte y almacenamiento durante su cadena de suministro, requieren un elevado grado de rigurosidad, debido a que existen problemas poscosecha ligados a factores como la temperatura y el tiempo de almacenamiento del ñame, los cuales influyen drásticamente en su tasa de respiración y de manera consecuente, en el crecimiento microbiano, deterioro biológico, transferencia de masa y la pérdida de peso del mismo (Andrade et al., 2012). Esta condición particular, se debe a que el material celular del tubérculo resulta susceptible al deterioro por el efecto de microorganismos, sumado al efecto catalizador del ambiente y a daños mecánicos durante el manejo (agrietamientos, cortes, roturas, abrasiones, perforaciones), que conllevan a perceptibles pérdidas de peso (Falade et al., 2007).

Por otro lado, suministrar condiciones apropiadas de almacenamiento para el ñame, contribuye a que sus tejidos amiláceos de alto contenido de humedad y tasa de transpiración moderada prolonguen su tiempo de vida útil y su calidad comercial ligada al uso y consumo (García et al., 2014). Por ello, cuando se tiene un centro de acopio para el almacenamiento de ñame, resulta indispensable disponer de una buena ventilación para evitar que la temperatura de almacenamiento se eleve drásticamente, esto con el propósito de impedir la exposición del producto a condiciones ambientales severas que modifiquen substancialmente sus condiciones de calidad (Holguín, 2011). Respecto a dichas condiciones de conservación o almacenamiento, a temperaturas bajas los tubérculos disminuyen su actividad metabólica y retrasan su periodo de reposo, asimismo, a temperaturas elevadas se presenta el envejecimiento gradual que se asocia al aumento de ácido 
abscísico (ABA) y a la reducción de ácido giberélico (GA) contenido en el producto. Lo anterior, también está relacionado con los tiempos de exposición específicos y la temperatura ambiente (Abdul et al., 2007).

Por otro lado, el uso de herramientas computacionales en el desarrollo de este tipo de estudios, reducen el tiempo de procesamiento de los datos, permitiendo obtener información de análisis con mayor precisión y eficiencia, para generar conclusiones con mayor exactitud y pertinencia a los requerimientos de cada investigación. Similarmente, resulta posible obtener respuesta a cada experimentación desarrollada con un mayor grado de certeza y con un nivel de experticia destacado. En este sentido, pueden referenciarse la utilización de herramientas de cómputo asociadas a entornos de desarrollo integrado (IDE) para el lenguaje de programación $\mathrm{R}$, con sofisticados complementos que posibilitan el análisis avanzado de datos a partir de la computación estadística y gráfica como la base interactiva de visualización, que puede ser utilizada para el estudio de fenómenos de transporte con fines prácticos, como es el caso de la trasferencia de masa del ñame (Tirado et al., 2014).

En vista a las persistentes complicaciones relacionadas con el manejo del ñame en actividades agrícolas, no solo en Colombia sino de manera generalizada, en los territorios dedicados a su producción; resulta imperante establecer medidas de estudio y diagnóstico que posibiliten la toma de decisiones prácticas en el área de conservación de este producto, con el fin de atribuir soluciones con mayor eficacia en las esferas sociales y económicas que se ven involucradas con el fenómeno de pérdida de peso del tubérculo mencionado. A razón de lo anterior, el objetivo de este estudio fue analizar mediante herramientas computacionales, la superficie de respuesta de la transferencia de masa durante la etapa de almacenamiento en la cadena de suministro de ñame (Dioscorea rotundata) tipo exportación.

\section{MATERIALES Y MÉTODOS}

Los aspectos metodológicos que se realizaron para el alcance del objetivo del estudio, se detallan de forma seguida.

\section{Obtención, disposición e identificación de muestras de ñame}

Las muestras de ñame (Dioscorea rotundata) se obtuvieron de un proceso de cultivo efectuado en el municipio de Sincé ubicado en el Departamento de Sucre; en un lapso de tiempo estimativo entre siete y diez meses, periodo durante el cual la cosecha posee la madurez fisiológica suficiente para ser extraída (Martínez y Moreno, 2012). La extracción, se realizó con la intervención de herramientas en materiales de madera y metálicos (palas, y picas) que posibilitaron la excavación del suelo sin causar daños relevantes al producto. Los rizomas obtenidos fueron lavados para retirar elementos físicos ajenos a la naturaleza del tubérculo tales como polvo, arena y suciedad en general; para luego ser recepcionados en el laboratorio de Bioprocesos de la Corporación Universitaria del Caribe (CECAR), ubicada en la carretera Troncal de Occidente, vía Corozal - Sincelejo (Sucre), instalaciones en las que se procedió a la selección de muestras. Esta etapa de selección, se llevó a cabo de manera aleatoria, asegurando la identificación de tubérculos con apariencia sana, específicamente en lo concerniente a las características organolépticas del ñame, (textura y color de la corteza, ausencia de anomalías físicas por efecto microbiano, etc.). Las muestras anteriormente mencionadas, fueron sometidas a una inspección y registro de peso inicial empleando una balanza de precisión. Una vez planteados los tratamientos de acuerdo a arreglos específicos de tiempo y temperatura, se procedió a la rotulación que se asignaría para cada muestra en particular y el almacenamiento en el desecador para evitar la pérdida de peso en el laboratorio hasta iniciar los procedimientos subsiguientes.

\section{Condiciones de almacenamiento}

Para el arreglo experimental, se evaluaron dos variables de incidencia, la temperatura de las condiciones ambientales en el experimento y el tiempo de almacenamiento, considerando múltiples tratamientos en cada una de estas variables, frente a una variable de interés o de respuesta, definida como el porcentaje de pérdida de peso del tubérculo (ecuación 1). Cabe destacar que, para mantener las temperaturas estipuladas para las muestras en la parte experimental, fue necesario emplear equipos de laboratorio que simularon estas condiciones ambientales. Estos equipos se especifican como, un refrigerador marca Imbera y modelo NV120, que opera con corriente de 115 voltios y equipado con un compresor de 1/6 HP; empleado para suministrar la temperatura de almacenamiento correspondiente a $\sim 10^{\circ} \mathrm{C}$. Una incubadora modelo IN-010 con potencia de calefacción de 350 Watts; empleada para suministrar la temperatura de almacenamiento correspondiente a $\sim 30^{\circ} \mathrm{C}$ y un esterilizador de aire caliente modelo YCO-010, en acero inoxidable, con una potencia de calefacción de 750 Watts; empleado para suministrar la temperatura de $\sim 35^{\circ} \mathrm{C}$. Estos últimos dos equipos, pertenecen a la marca Gemmy Industrial Corp. La otra opción de almacenamiento evaluada fue a temperatura ambiente del laboratorio $\left(\sim 32.5^{\circ} \mathrm{C}\right)$, sin emplear algún equipo o empaque en especial. 


\section{Arreglo experimental}

Para detallar el efecto que tuvo la temperatura en el experimento y el tiempo de almacenamiento sobre la transferencia de masa o pérdida de peso del ñame durante la etapa de almacenamiento, las muestras de ñame se sometieron a diversas condiciones de temperatura, específicamente a $10^{\circ} \mathrm{C}, 30^{\circ} \mathrm{C}, 32.5^{\circ} \mathrm{C}$ y $35^{\circ} \mathrm{C}$; con distintos tiempos de almacenamiento, que corresponden a 10, 20, 30, 40, 50 y 60 días. Se consideraron estos tiempos con el propósito de observar el comportamiento de la variable de interés del estudio, es decir, la pérdida de peso que experimentó el tubérculo. El cálculo de la pérdida de peso se expresó en porcentaje y se determinó mediante el uso de la ecuación 1.

$$
\% p p=\frac{(p i-p f)}{p i} \times 100
$$

Donde, el \%pp es el porcentaje de pérdida de peso en el producto, pi es el peso inicial del producto y pf es el peso final del producto.

\section{Análisis estadístico de los datos}

Para el análisis estadístico de los datos, se utilizó un experimento factorial bajo arreglo completamente al azar, por triplicado, donde los factores fueron la temperatura con cuatro niveles $\left(10^{\circ} \mathrm{C}, 30^{\circ} \mathrm{C}, 32.5^{\circ} \mathrm{C}\right.$ y $\left.35^{\circ} \mathrm{C}\right)$ y el tiempo de almacenamiento con seis niveles (10d, $20 \mathrm{~d}, 30 \mathrm{~d}, 40 \mathrm{~d}, 50 \mathrm{~d}$ y $60 \mathrm{~d})$. El modelo matemático que se utilizó para el experimento factorial, se describe en la ecuación 2.

$$
y_{i j k}=\mu+\alpha_{j}+\beta_{k}+(\alpha \beta)_{j k}+\varepsilon_{i j k}
$$

Donde, $y_{i j k}$ es la variable respuesta, en este caso pérdida de peso en la i-ésima unidad experimental, para el j-ésimo nivel de la temperatura de almacenamiento, del k-ésimo nivel del factor tiempo de almacenamiento, $\mu$ es la media general de los tratamientos, $\alpha_{j}$ es el efecto principal del j-ésimo nivel del factor temperatura de almacenamiento, $\beta_{k}$ es el efecto principal del k-ésimo nivel del factor tiempo de almacenamiento, $(\alpha \beta)_{j k}$ es el efecto del j-ésimo nivel del factor temperatura de almacenamiento, con el efecto del k-ésimo del nivel del factor tiempo de almacenamiento, $\varepsilon_{i j k}$ es el error aleatorio en la i-ésima unidad experimental para el j-ésimo nivel del factor temperatura de almacenamiento y k-ésimo nivel del factor tiempo de almacenamiento. Adicionalmente, para establecer el efecto de las variables independientes relacionadas con la temperatura y el tiempo de almacenamiento sobre la variable dependiente de interés que fue la pérdida de peso durante el almacenamiento por ocasión de la transferencia de masa, se aplicó un análisis de varianza ANOVA ( $\leq \leq 0.05$ ), para comparar los valores medios e identificar la prevalencia de diferencias significativas para la pérdida de peso de las muestras de ñame en función de los tratamientos efectuados.

De forma complementaria a este análisis, se utilizó la prueba de rangos múltiples de Tukey $(p \leq 0.05)$ para aquellas variables que mostraron diferencias significativas de acuerdo con el factor de variación aplicado y de esa forma obtener detalladamente la respuesta que permitiera precisar los tratamientos que mostraron diferencias significativas en el experimento. Debe mencionarse, que para efectuar este último análisis los factores empleados se consideraron como cuantitativos para detallar el comportamiento matemático de cada interacción entre los niveles de los factores estudiados.

\section{Algoritmo computacional de optimización}

Para la obtención del análisis computacional que permita graficar mediante una superficie de respuesta, la pérdida de peso del ñame durante su etapa de almacenamiento, se utilizó un algoritmo de optimización de gradiente descendiente. Este método inicia con un punto $x^{0}$ y se va modificando el vector de decisión de forma iterativa, hasta alcanzar el número Niter; el cual corresponde al número de iteraciones posibles para hallar la solución óptima $x^{*}$ (Jiménez et al., 2016). El modelo matemático utilizado para el método de gradiente descendiente es el que se ilustra en la ecuación 3.

$$
x^{k+1}=x^{k}-\alpha \cdot g(f(x))
$$

Donde, $k$ es la iteración actual y $\propto$ notoriamente es el paso de búsqueda empleado $(\propto=0.05), g(f(x))$ es el gradiente de la función $f(x)$. Para este caso en particular, el interés fue determinar el tratamiento que minimizara la pérdida de peso en el ñame, por lo tanto fue necesario considerar que la dirección de descenso debe obtenerse en sentido contrario a $g$ es decir, multiplicar por (-1) para así garantizar que $f\left(x^{k+1}\right)<f\left(x^{k}\right)$, lo que sin duda implicaría que la solución nueva que se generara fuera menor a la inmediatamente anterior. 


\section{RESULTADOS Y DISCUSIÓN}

A partir del análisis de varianza, fue posible confirmar la influencia de la temperatura y del tiempo de almacenamiento sobre la pérdida de peso de las muestras de ñame. La Tabla 1, indica los resultados obtenidos en este análisis, a través de la cual es posible argüir que cuando los factores de tiempo y temperatura de almacenamiento actúan de forma conjunta, generan diferencias significativas en la variable de respuesta. Este resultado se atribuye a que los tratamientos efectuados representan tasas de diferenciación en los mecanismos de flujo de masa atribuidos a la fuerza impulsora a la que fueron sometidas las muestras, por tanto, los tratamientos aplicados representan una influencia notoria sobre el tubérculo, lo cual indica que las condiciones ambientales de almacenamiento suministradas posibilitan la obtención de diferencias significativas en la pérdida de peso del ñame fresco, cuando actúan de forma conjunta la temperatura y el tiempo de almacenamiento (Paul et al., 2019; Otegbayo, 2014). De igual forma, de este análisis se obtuvo que los factores temperatura de las condiciones ambientales y tiempo de almacenamiento actuando de forma independiente también resultan tener un efecto significativo sobre los valores medios de la pérdida de peso, lo cual es congruente con los resultados obtenidos para el análisis de varianza del proceso de secado del ñame (Montes et al., 2008).

Dados los resultados anteriores y confirmada la significancia de la interacción Temperatura $\left({ }^{\circ} \mathrm{C}\right){ }^{*}$ Tiempo (d) sobre la variable de respuesta, en la Tabla 2 se ilustra la interacción derivada de la influencia del tiempo dentro de cada nivel de temperatura, a partir de las cuales se obtuvieron los modelos matemáticos específicos para cada caso. Sin embargo, para determinar la significancia de dichos modelos, fue necesario llevar a cabo un nuevo análisis de varianza; en el que inicialmente se evaluó la temperatura actuando de manera independiente mediante el test de comparaciones múltiples de Tukey para factores cuantitativos, se observó que su valor-p no supera el $5 \%$, siendo un resultado congruente con el análisis de varianza inicial de la Tabla 1.

Tabla 1: Resultados del análisis de varianza obtenido

\begin{tabular}{|l|l|l|l|l|l|}
\hline Factores & $G L$ & $S C$ & $C M$ & $F C$ & Valor-P \\
\hline Temperatura $\left({ }^{\circ} \mathrm{C}\right)$ & 3 & 560.22 & 186.74 & 51.67 & 0.00 \\
\hline Tiempo $(\mathrm{d})$ & 5 & 642.39 & 128.47 & 35.55 & 0.00 \\
\hline Temperatura $\left({ }^{\circ} \mathrm{C}\right)^{\star}$ Tiempo $(\mathrm{d})$ & 15 & 139.63 & 9.30 & 2.57 & 0.00 \\
\hline Error & 48 & 173.45 & 3.61 & & \\
\hline Total & 71 & 1515.68 & & & \\
\hline
\end{tabular}

De este nuevo análisis (Tabla 2), se resalta que la interacción del factor tiempo con el nivel de $10^{\circ} \mathrm{C}$ de temperatura no resultó ser significativo para la pérdida de peso del ñame, lo cual se atribuye a que el contacto de la matriz porosa del ñame con el aire frio proveniente de equipo de refrigeración, no genera un decremento significativo de peso del tubérculo, puesto que el empleo de este tipo de temperaturas en el almacenamiento reducen la actividad enzimática de los alimentos, disminuyendo así su actividad biológica, el crecimiento de microrganismos y ocasionándose así la disminución del contenido de humedad por fenómenos de transporte (Inestroza-Lizardo et al., 2016). Es contraste, todas las demás interacciones del tiempo con los niveles de temperaturas de $30^{\circ} \mathrm{C}$, $32.5^{\circ} \mathrm{C}$ y $35^{\circ} \mathrm{C}$, si resultaron ser significativas para la pérdida de peso del ñame durante la etapa de almacenamiento. Este fenómeno es de esperarse, ya que la transferencia de masa se regula por los fenómenos fisiológicos de respiración celular de las matrices porosas, como es el caso del ñame, teniendo la temperatura en función del tiempo un papel fundamental en este suceso (Martínez-González et al., 2017).

Tabla 2: Análisis de varianza del factor tiempo de almacenamiento (d) dentro de cada nivel del factor temperatura $\left({ }^{\circ} \mathrm{C}\right)$

\begin{tabular}{|l|l|l|l|l|l|}
\hline Factores e interacciones & GL & SC & $C M$ & $F C$ & Valor-P \\
\hline Temperatura $\left({ }^{\circ} \mathrm{C}\right)$ & 3 & 560.22 & 186.74 & 51.67 & 0.00 \\
\hline Tiempo (d): Temperatura $\left({ }^{\circ} \mathrm{C}\right) 10$ & 5 & 8.54 & 1.70 & 0.47 & 0.79 \\
\hline Tiempo (d): Temperatura $\left({ }^{\circ} \mathrm{C}\right) 30$ & 5 & 182.58 & 36.51 & 10.10 & 0.00 \\
\hline Tiempo (d): Temperatura $\left({ }^{\circ} \mathrm{C}\right) 32.5$ & 5 & 306.75 & 61.35 & 16.97 & 0.00 \\
\hline Tiempo (d): Temperatura $\left({ }^{\circ} \mathrm{C}\right) 35$ & 5 & 284.11 & 56.82 & 15.72 & 0.00 \\
\hline Error & 48 & 173.44 & 3.61 & & \\
\hline Total & 71 & 1515.67 & & & \\
\hline
\end{tabular}

Luego de obtener los resultados que indican la relevancia de las interacciones analizadas, se procedió a definir el modelo matemático que sigue cada una de las condiciones de almacenamiento y las temperaturas ambientales de $30^{\circ} \mathrm{C}, 32.2^{\circ} \mathrm{C}$ y $35^{\circ} \mathrm{C}$, empleadas en el experimento. Tal modelo matemático, permite definir el comportamiento de la pérdida de peso, dadas esas condiciones de almacenamiento específicas. Los modelos matemáticos obtenidos se encuentran descritos en la Tabla 3, así como la síntesis del comportamiento del factor tiempo, dentro 
de los niveles de temperatura. Como se había mencionado anteriormente, para la temperatura de $10^{\circ} \mathrm{C}$, se obtuvo que el modelo no es significativo, por lo que no afecta notoriamente la pérdida de peso. A temperaturas de $30^{\circ} \mathrm{C}$, $32.5^{\circ} \mathrm{C}$ y $35^{\circ} \mathrm{C}$, se obtuvieron modelos matemáticos de tipo lineal, donde y representa la pérdida de peso, seguido del valor numérico de intercepto, más el valor numérico de la pendiente $x$, que es la variación en el factor tiempo de almacenamiento.

En este caso, por ejemplo, el modelo de la temperatura a $30^{\circ} \mathrm{C}$, posee el intercepto en 0.54 y pendiente positiva de 0.18. Además de la designación de los modelos, existe un coeficiente de determinación $\left(R^{2}\right)$ que indica la proporción de la variación de los resultados de la pérdida de peso que se explica por el modelo matemático obtenido, es decir, este indicador determina la calidad del modelo matemático obtenido para lograr replicar satisfactoriamente los resultados de la pérdida de peso en el tubérculo en función del tiempo de almacenamiento (Lavalle et al., 2006). Según los datos obtenidos, el modelo representa un $98 \%$ esta condición, lo cual es considerado un buen ajuste del modelo a las condiciones fenomenológicas experimentadas para el almacenamiento del ñame. La significancia del modelo (Valor-p) claramente es adecuada, debido a que posee un valor de cero (0), de igual forma, se evidencia que no existe una falta de ajuste, al ser este valor mayor al nivel de significancia preestablecido para el estudio.

Tabla 3: Resumen del comportamiento del factor tiempo de almacenamiento dentro de los niveles del factor temperatura.

\begin{tabular}{|l|l|l|l|l|}
\hline Nivel del factor & Modelo & $R^{2}$ & $P$-valor & Falta de ajuste \\
\hline Temperatura $10^{\circ} \mathrm{C}$ & No significativo & No significativo & No significativo & No significativo \\
\hline Temperatura $30^{\circ} \mathrm{C}$ & $y=0.5411+0.1854 x$ & 0.98 & 0.00 & 0.96 \\
\hline Temperatura $32.5^{\circ} \mathrm{C}$ & $\mathrm{y}=-1.0418+0.2209 \mathrm{x}$ & 0.98 & 0.00 & 0.86 \\
\hline Temperatura $35^{\circ} \mathrm{C}$ & $\mathrm{y}=0.2651+0.2397 \mathrm{x}$ & 0.98 & 0.00 & 0.84 \\
\hline
\end{tabular}

De forma complementaria, es de resaltar que el valor de la pendiente $\left(b_{0}\right)$ para el modelo matemático obtenido con el nivel de temperatura de $35^{\circ} \mathrm{C}$ fue superior en comparación con los otros tratamientos suministrados, lo cual indica que el grado de inclinación de este modelo es mayor y por ende las estimaciones que se realicen con su empleo indicarán una mayor pérdida de peso durante el almacenamiento del ñame, lo cual es totalmente congruente con los fenómenos de transporte que indican que a mayor temperatura sobre las matrices vegetales porosas se obtendrá una mayor pérdida de peso hasta alcanzar el estado de equilibrio (Cai et al., 2015; Kumar et al., 2014; Perussello et al., 2014). De esta misma forma, una vez realizada la valoración teniendo en cuenta cada nivel de temperatura; se llevó a cabo una comparación similar, pero esta vez, se procedió a analizar la interacción derivada de la influencia de la temperatura de las condiciones ambientales dentro de cada nivel de tiempo de almacenamiento a través de un análisis de varianza, lo cual es congruente metodológicamente con los experimentos factoriales, lo resultados obtenidos se registran en la Tabla 4.

Tabla 4: Análisis de varianza de la temperatura $\left({ }^{\circ} \mathrm{C}\right)$ dentro de cada nivel de tiempo $(\mathrm{s})$

\begin{tabular}{|l|l|l|l|l|l|}
\hline Factores e interacciones & GL & SC & CM & FC & Valor-P \\
\hline Tiempo (d) & 5 & 642.38 & 128.47 & 35.55 & 0.00 \\
\hline Temperatura $\left({ }^{\circ} \mathrm{C}\right):$ Tiempo (d) 10 & 3 & 9.68 & 3.22 & 0.89 & 0.45 \\
\hline Temperatura $\left({ }^{\circ} \mathrm{C}\right):$ Tiempo (d) 20 & 3 & 30.35 & 10.11 & 2.80 & 0.04 \\
\hline Temperatura $\left({ }^{\circ} \mathrm{C}\right):$ Tiempo (d) 30 & 3 & 65.00 & 21.66 & 5.99 & 0.00 \\
\hline Temperatura $\left({ }^{\circ} \mathrm{C}\right):$ Tiempo (d) 40 & 3 & 132.08 & 44.02 & 12.18 & 0.00 \\
\hline Temperatura $\left({ }^{\circ} \mathrm{C}\right):$ Tiempo (d) 50 & 3 & 218.70 & 72.90 & 20.17 & 0.00 \\
\hline Temperatura $\left({ }^{\circ} \mathrm{C}\right):$ Tiempo (d) 60 & 3 & 244.00 & 81.33 & 22.50 & 0.00 \\
\hline Error & 48 & 173.44 & 3.61 & & \\
\hline Total & 71 & 1515.67 & & & \\
\end{tabular}

Al analizar el tiempo de almacenamiento, actuando de manera independiente, se observa que este factor ocasiona diferencias significativas en los valores medios de pérdida de peso del ñame, lo cual es un resultado congruente con el análisis de varianza inicial. Adicionalmente, en todas las interacciones de tiempo de almacenamiento y de temperatura a excepción de la interacción temperatura $\left({ }^{\circ} \mathrm{C}\right)$ : tiempo 10 días, se obtuvieron interacciones significativas para los valores medios de la pérdida de peso del tubérculo. Por tanto, se afirma que un tiempo de almacenamiento igual a 10 días, bajo cualquier condición de temperatura ambiental no representará una pérdida de peso notoria en el ñame, lo cual es un resultado favorable para los productores y comercializadores de este tubérculo, puesto que su valor de comercialización en el mercado depende estrictamente del peso en libras o kilogramos que contenga el producto. Por ello, determinar que en los primeros 10 días de almacenamiento las pérdidas de peso no son significativas, favorece a las actividades de planificación y gestión a lo largo de la cadena de suministro para sus involucrados (Allaoui et al., 2019). En complemento a lo anterior, también se obtuvieron modelos matemáticos que describen el comportamiento del factor temperatura, 
dentro de los niveles de tiempo de almacenamiento de 20, 30, 40, 50 y 60 días, respectivamente. El resumen de estos resultados, se indican en la Tabla 5, y como se había citado previamente, en las interacciones del factor tiempo dentro de los niveles de temperatura, se describen modelos de tipo lineal y pendiente positiva para todas las interacciones.

Tabla 5: Resumen del comportamiento del factor Temperatura dentro de los niveles del factor Tiempo.

\begin{tabular}{|l|l|l|l|l|}
\hline Nivel del factor & Modelo & $R^{2}$ & $P$-valor & Falta de ajuste \\
\hline Tiempo $10 \mathrm{~d}$ & No significativo & No significativo & No significativo & No significativo \\
\hline Tiempo $20 \mathrm{~d}$ & $\mathrm{y}=-0.8938+0.1506 \mathrm{x}$ & 0.87 & 0.00 & 0.60 \\
\hline Tiempo $30 \mathrm{~d}$ & $\mathrm{y}=-1.4115+0.2331 \mathrm{x}$ & 0.98 & 0.00 & 0.86 \\
\hline Tiempo $40 \mathrm{~d}$ & $\mathrm{y}=-0.1972+0.3343 \mathrm{x}$ & 0.99 & 0.00 & 0.92 \\
\hline Tiempo 50d & $\mathrm{y}=-2.4784+0.4309 \mathrm{x}$ & 0.99 & 0.00 & 0.96 \\
\hline Tiempo 60d & $\mathrm{y}=-2.2224+0.4547 \mathrm{x}$ & 0.99 & 0.00 & 0.90 \\
\hline
\end{tabular}

Según la Tabla 5, a medida que se incrementó el tiempo de almacenamiento del ñame, también se obtuvo un aumento en los valores de las pendientes de los modelos, lo que invita a deducir que existe una relación directa entre el tiempo de almacenamiento y la pérdida de peso del tubérculo, siendo mucho más pronunciada la reducción de peso en el producto a medida que el tiempo de almacenamiento transcurre. Resultados similares a estos han sido analizados en tubérculos y hortalizas como la batata y la ahuyama, concretando que almacenar estos productos por prolongados periodos de tiempo no genera reducciones significativas en su contenido nutricional, pero los procesos de respiración acelerada provocan reducciones de peso en los productos. De igual forma, los modelos obtenidos tienen buenos referentes al coeficiente de determinación, lo cual indica que pueden ser empleados para estimar resultados de pérdida de peso en función de las condiciones de almacenamiento (Zaccari et al., 2017).

A partir de los resultados anteriores, fue posible desarrollar mediante herramientas software, la representación esquemática del comportamiento de la superficie de respuesta de la transferencia de masa. La Figura 1, corresponde con tales resultados, e ilustra el comportamiento de la pérdida de peso frente a las condiciones de conservación del ñame que han sido descritas. Así mismo, es posible visualizar que, a medida que la temperatura y el tiempo de almacenamiento se incrementan, igualmente se presenta un mayor porcentaje de pérdida de peso en las muestras de ñame que se procesaron, por lo que resulta posible afirmar que el efecto ocasionado por la fuerza impulsora derivada de la configuración de temperatura y del tiempo empleados, es notable; es decir, que el diferencial de temperatura que se genera entre la superficie externa del ñame con las condiciones de temperatura del medio, trae consigo un impulso capaz de ocasionar mayor transferencia de humedad entre el producto y su medio circundante, resultando en su pérdida de peso. Adicionalmente, la pérdida de peso en el producto también está condicionada por la cantidad de tiempo a la que este se exponga a dichas condiciones de almacenamiento, estableciendo una relación directa entre ellos, es decir, entre más prolongado sea el tiempo de almacenamiento, menor peso tendrá el producto final, lo cual representa un efecto económico negativo en los productores del tubérculo, dado que con la pérdida de peso del ñame se compromete el margen neto de contribución que genera el producto durante las actividades de comercialización y con ello las utilidades económicas que se derivan de esta actividad (Rodríguez-Manrique et al., 2018; Moreno y Bouchon, 2008).

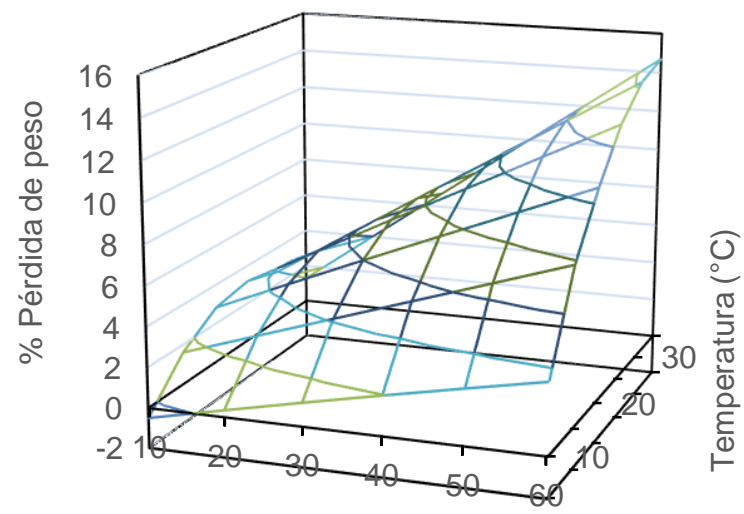

Tiempo (d)

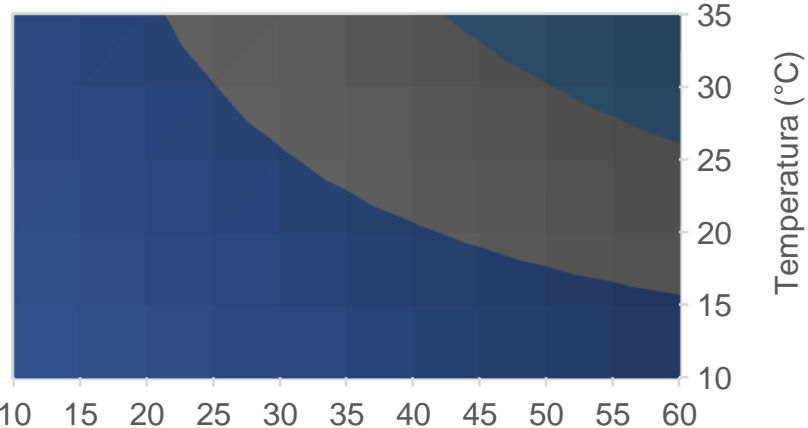

Tiempo $(\mathrm{d})$

$\square-5 \quad$ - 5-10 $\square$ 10-15

Fig. 1: Superficie de respuesta de la pérdida de peso del ñame bajo diversas condiciones de almacenamiento.

Según los resultados obtenidos, el modelo matemático al cual se han ajustado los datos para modelar todo el proceso de la pérdida de peso del ñame durante la etapa de almacenamiento, es ilustrado en la ecuación 4. 


$$
\% p p=-0,490017+0,0308497 T-0,032572 t-0,000664463 T^{2}+0,00798115 T t-0,000113095 t^{2}
$$

Donde, $T$ es el efecto de la temperatura de las condiciones ambientales y $t$ es el efecto del tiempo de almacenamiento. Debe resaltarse que el modelo obtenido (ecuación 4) para la superficie de respuesta de la pérdida de peso del ñame, fue utilizado por el algoritmo de gradiente descendiente para buscar el punto óptimo que minimizara la pérdida de peso en función de la variación de los niveles de temperatura y del tiempo de almacenamiento empleados de forma computacional; además, bajo la consideración de que el modelo obtenido y representado en la Figura 1, presentaba mínimos locales que pudieran representar obstáculos para el algoritmo empleado. Entonces, fue posible corroborar gráficamente el ajuste de*l modelo mediante el uso del algoritmo, los resultados obtenidos se ilustran en la Figura 2.

a)



$\square 0-5 \square 5-10 \square 10-15$ b)

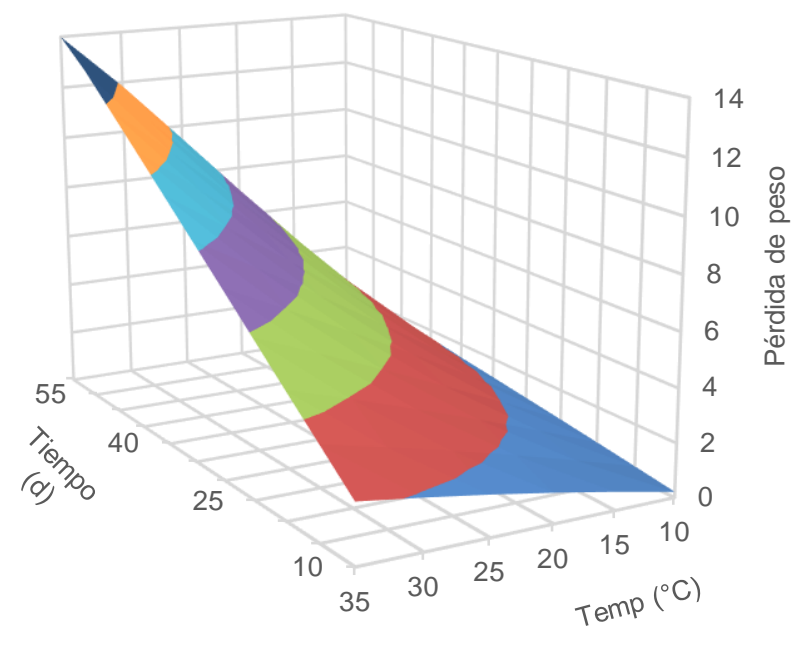

=0-2 $=2-4=4-6 \quad 6-8 \quad \square-10 \quad 10-12 \quad \square 12-14$

Fig. 2: a) Contorno obtenido mediante el empleo del método de optimización del algoritmo de gradiente descendiente; b) Superficie de respuesta obtenida mediante el empleo del método de optimización del algoritmo de gradiente descendiente.

Como es de observarse en la Figura 2, el resultado de la minimización indica que los valores más bajos de la pérdida de peso se alcanzan a menor tiempo (10 días) de almacenamiento y menor temperatura de las condiciones ambientales $\left(10^{\circ} \mathrm{C}\right)$, lo cual es coherente con los resultados del diseño experimental. Por tanto, los algoritmos de gradiente descendiente pueden ser una técnica computacional eficiente para determinar los puntos mínimos de situaciones donde los modelos experimentales sean doblemente derivables y unimodales, es decir, que tengan un valor mínimo general libre de mínimos locales, como es el caso que se obtuvo en este estudio para la transferencia de masa del ñame. En estudios similares donde se examinó la influencia de la temperatura y el tiempo de almacenamiento en la conservación del mango, también se referencia que el análisis de varianza indica una interacción altamente significativa entre la temperatura y el tiempo de almacenamiento. Por lo cual, cada nivel de temperatura fue analizado de manera separada (Galvis et al., 2002). En todas las temperaturas de almacenamiento, el porcentaje de pérdida de peso de los frutos aumentó con el tiempo de almacenamiento. Este comportamiento también es aplicable a lo afirmado por Wills (1998), quien afirma que, a mayor temperatura mayor, intensidad respiratoria y velocidad de transpiración me tejidos vegetales, por ende, mayor tasa de transferencia de masa.

\section{DISCUSIÓN FINAL}

El uso de herramientas computacionales con complementos de análisis estadístico de datos y representación gráfica, como la base interactiva de visualización, permitió determinar de manera detallada y precisa, la forma en que la temperatura de las condiciones ambientales y el tiempo de almacenamiento presentan una influencia significativa sobre la pérdida de peso del ñame tipo exportación, lo cual resulta valioso como fuente de información para los involucrados en la actividad productiva, sobre todo para el desarrollo de actividades de planificación y gestión a lo largo de la cadena de suministro del tubérculo. De los resultados y respectivos análisis de este estudio, fue posible definir que entre mayor sea la temperatura de las condiciones ambientales y tiempo de almacenamiento, mayor será el registro del porcentaje de pérdida de peso en el producto de naturaleza vegetal, esto debido a que existe un aumento en el efecto de la fuerza impulsora en matrices porosas como el ñame, la cual estimula la transferencia de masa en el tubérculo. En este caso, la fuerza impulsadora se fundamenta entre 
la diferencia de temperaturas entre las superficies de los cuerpos involucrados, provocando así que la superficie con más baja temperatura, es decir, el tubérculo, reciba energía de la superficie con más alta temperatura, que se concentra en el ambiente de almacenamiento y, por consiguiente, pierda peso conforme transcurre el tiempo y la fuerza impulsora aumenta. En caso contrario, tiempos de almacenamiento de 10 días y temperaturas de condiciones ambientales de $10^{\circ} \mathrm{C}$ no representan cambios significativos en la variable de estudio, sin embargo, el almacenamiento del ñame en condiciones de refrigeración genera consecuencias desfavorables sobre aspectos de calidad del producto, ratificándose daños colaterales por frio que se observaron durante la experimentación. Por último, el uso de algoritmos computacionales como el caso del gradiente descendiente, pueden considerarse como una herramienta para optimizar fenómenos de transporte relacionados con la pérdida de peso de matrices porosas, ya que esta técnica permite determinar de forma eficiente los valores mínimos de la variable de interés en este tipo de estudios.

\section{CONCLUSIONES}

De acuerdo al trabajo presentado y a los resultados obtenidos, se pueden plantear las siguientes conclusiones principales:

1. El método empleado para el estudio de la transferencia de ñame es práctico y reproducible, permite obtener resultados pertinentes a los fenómenos de transferencia de masa de matrices alimentarias.

2. La característica principal de la metodología empleada, radica en los análisis estadísticos acompañados de algoritmos computacionales que permiten obtener deducciones apropiadas al estudio de la transferencia de masa de matrices porosas, para la toma de decisiones de las actividades productivas de las cadenas agroalimentarias.

3. Las herramientas computacionales empleadas para el estudio de la transferencia de masa del ñame presentan alta congruencia, practicidad y exactitud con los resultados obtenidos en el diseño estadístico.

\section{REFERENCIAS}

Abdul, J. C., Gopi, R., y otros cuatro autores, Changes in biochemical constituents and induction of early sprouting by triadimefon treatment in white yam (Dioscórea rotundata Poir.) tubers during storage, doi: 10.1631/jzus. 2007.B0283, J. Zhejiang Univ Sci. B., 8 (4), 283 - 288 (2007)

Acevedo, A., Salcedo, J., y Sandoval, I., Desarrollo y productividad de ñame (Dioscorea tríifa) y (Dioscorea esculenta) en diferentes condiciones hídricas, doi: 10.15446/acag. v64n1.43917, Rev. Acta Agronómica, 64(1), 30- 35 (2015)

Acosta, A., y Blanco, C., Obtención y caracterización de almidones nativos colombianos para su evaluación como posibles alternativas en la industria alimentaria. Universidad de Cartagena. Facultad de Ingeniería. Cartagena de Indias, (2013)

Agronet, Exportadores de nombre de la mano del ICA. Ministerio de Agricultura y Desarrollo Rural, disponible en: https://www.agronet.gov.co/Noticias/Paginas/Noticia241.aspx. (2009)

Allaoui, H., Guo, Y., y Sarkis, J., Decision support for collaboration planning in sustainable supply chains, doi: 10.1016/j.jclepro.2019.04.367, J. of Cleaner Production, 229, 761-774 (2019)

Alvis, A., Rodríguez, J., y Arrázola, G., Efecto del freído sobre cambios de humedad y absorción de aceite en ñame (Dioscórea rotundata P), Rev. Agronomía Colombiana, ISSN: 2539-4142, 34 (1), (2016)

Andrade, R., Palacio, J., Pacheco, W., y Betin, R., Almacenamiento de Trozos de Name (Dioscórea rotundata Poir) en Atmósferas Modificadas, doi: 10.4067/S0718-07642012000400008, Inf. Tecnol., 23(4), 65-72 (2012)

Cai, W., Zhu, L., y otros tres autores, Effect of thermophysical properties on coupled heat and mass transfer in porous material during forced convective drying, doi: 10.1155/2014/830387, J. Advances in Mechanical Engineering, 6, 830387 (2015)

Yan, D., y Lin, J., Effects of different storage conditions on steroidal saponins in yam (Dioscorea pseudojaponica Yamamoto) tubers. Food Chemistry, 110 (2008) 670-677, (2008)

Falade, K., Olurin, T., y otros dos autores, Effect of pretreatment and temperature on air-drying of Dioscoréa alata and Dioscoréa rotundata slices, doi: 10.1016/j.jfoodeng.2006.06.034, J. of Food Engineering, 80(4), 1002-1010 (2007)

Zaccari, F., Cabrera, M., and Saadoun, A., Glucose Content and In Vitro Bioaccessibility in Sweet Potato and Winter Squash Varieties during Storage, doi: 10.3390/foods6070048, J. Foods, Foods 2017, 6(7), 48 (2017)

Galvis, J., Arjona, H., y otros tres autores, Influencia de la temperatura y el tiempo de almacenamiento en la conservación del fruto de mango (Manifera Indica I.) variedad Van Dyke, Rev. Agronomía Colombiana, ISSN: 2357-3732, 19 (1-2), 2336 (2002)

García, A., Pérez, M., y García, A., Evaluación del comportamiento postcosecha de la batata (Ipomea batatas (L) Lam) en condiciones de almacenamiento comercial, Rev. Iberoamericana de Tecnología Postcosecha, ISSN: 1665-0204, 15 (2), 177-186 (2014) 
King, G., A Systems approach to improving subsistence yam production in Central Province, Papua New Guinea, Agricultural Systems, ISSN 0308-521X, https://doi.org/10.1016/0308-521X(89)90018-8. Volumen 31, Issue 2, 1989, Pages 157-168 (1989)

González, M., El Ñame (Dioscórea spp.). Características, usos y valor medicinal. Aspectos de importancia en el desarrollo de su cultivo, Rev. Cultivos Tropicales, ISSN: 0258-5936, 33(4), 05-15 (2012)

Holguín, M., y Mercado, Y., Análisis bromatológico del tubérculo seco y pulverizado de Dioscórea cayenensis Ñame amarillo-Universidad de Sucre. Facultad de Ciencias y Educación. Sincelejo, (2011)

Inestroza-Lizardo, C., Voigt, V., y otros dos autores, Métodos de enfriamiento aplicables a frutas y hortalizas enteras y mínimamente procesadas. Rev. Iberoamericana de Tecnología Postcosecha, ISSN: 1665-0204, 17(2), 149-161 (2016)

Cuevas, E., Oliva, D., Díaz, M., y Osuna, J., Optimización: algoritmos programados con MATLAB. 1ª edición, 171 - 190. Alfa omega, México D.C (2016)

Kumar, C., Karim, A., y Joardder, M., Intermittent drying of food products: A critical review, doi: 10.1016/j.jfoodeng.2013.08.014, J. of Food Engineering, 121, 48-57 (2014)

Lavalle, A., Micheli, E., y Rubio, N., Análisis didáctico de regresión y correlación para la enseñanza media, Rev. Latinoamericana de investigación en matemática educativa, ISSN: 2007-6819, 9(3), 383-406 (2006)

Martínez-González, M., Balois, R., y otros cuatro autores. Poscosecha de frutos: maduración y cambios bioquímicos, doi: 10.29312/remexca.v0i19.674, Rev. Mexicana de ciencias agrícolas, 19, 4075-4087 (2017)

Montes, E., Gallo, R., y otros cuatro autores, Modelado de la cinética de secado de ñame (dioscórea rotundata) en capa delgada. Rev. Ingeniería e investigación, ISSN: 0120-5609, 28(2), 45-52 (2008)

Moreno, M., y Bouchon, P., A different perspective to study the effect of freeze, air, and osmotic drying on oil absorption during potato frying, doi: 10.1111/j.1750-3841.2008.00669.x, J. of food science, 73(3), E122-E128 (2008)

Onyeka, T., Pétro, D., y otros tres autores, Resistance in water yam (Dioscorea alata) cultivars in the French West Indies to anthracnose disease based on tissue culture-derived whole-plant assay, doi: 10.1111/j.1365-3059.2006.01436.x, J. Plant Pathology, 55(5), 671-678, (2006)

Otegbayo, B., Effect of storage on the pasting characteristics of yam tubers, doi: 10.1016/S0189-7241(15)30126-0, Nigerian Food Journal, 32(2), 113-119 (2014)

Guillou, P., Marc, O., y otros tres autores, Heat and mass transfer local modelling applied to biomass briquette drying, doi: 10.1016/j.promfg.2019.05.018, J. Procedia Manufacturing, 35, 149-154 (2019)

Perussello, C., Kumar, C., De Castilhos, F., y Karim, M., Heat and mass transfer modeling of the osmo-convective drying of yacon roots (Smallanthus sonchifolius), doi: 10.1016/j.applthermaleng.2013.10.020, J. Applied Thermal Engineering, 63(1), 23-32 (2014)

Reina-Aranza, Y., El cultivo de ñame en el Caribe colombiano, Documentos de Trabajo sobre Economía Regional, ISSN: 1692-3715, 2012, no. 168 (2012)

Rodríguez-Manrique, J., Alvis-Bermúdez, A., y Cohen-Manrique, C., Análisis de Perfil de Textura de Ahuyama (Cucurbita maxima) sometida a Freído Atmosférico por Inmersión, Información tecnológica, ISSN: 0718-0764, 29(4), 55-64 (2018)

Tamiru, M., Becker, H., y Maass, B., Diversity, distribution and management of yam landraces (Dioscorea spp.) in Southern Ethiopia, doi: 10.1007/s10722-007-9219-4, J. Genetic Resources and Crop Evolution, 55(1), 115-131 (2008)

Tirado, D., Acevedo, D., y Puello, P., Determinación computacional del coeficiente de transferencia de calor y propiedades termofísicas de alimentos, doi: 10.4067/S0718-07642014000300008, Información tecnológica, 25(3), 53-58 (2014)

Wills, R., Introducción a la fisiología y manipulación de frutas y hortalizas. $2^{a}$ edición, 18- 41. Acribia S.A. Zaragoza, España (1998)

Chen, Y., y Lin, K., Effects of heating temperature on the total phenolic compound, antioxidative ability and the stability of dioscorin of various yam cultivars. Food Chemistry, 101 (2007) 955-963, (2006) 